\title{
Spatial differences and driving forces of land urbanization in China
}

\author{
LIN Xueqin", "WANG Yang ${ }^{2,3}$, WANG Shaojian ${ }^{4,5}$, WANG Dai ${ }^{4}$ \\ 1. College of Resources Environment and Tourism, Capital Normal University, Beijing 100048, China; \\ 2. Guangzhou Institute of Geography, Guangzhou 510070, China; \\ 3. Guangdong Open Laboratory of Geospatial Information Technology and Application, Guangzhou 510070 \\ China; \\ 4. Institute of Geographic Sciences and Natural Resources Research, CAS, Beijing 100101, China; \\ 5. University of Chinese Academy of Sciences, Beijing 100049, China
}

\begin{abstract}
Land urbanization plays an important supporting and restriction role in the rapid and sustainable development of urbanization in China, and it shows distinctive spatial heterogeneity. Applying urban area as the basic research unit and urban construction land area as the core indicator, this paper establishes the conceptual framework and calculation method for the quantity and rate of land urbanization process. The study evaluates the spatial differentiation pattern of absolute and relative process of land urbanization in 658 cities in China from 2000 to 2010. The spatial distribution of cities with rapid land urbanization process is discussed, and the contribution rate and its spatial heterogeneity of major land use types are examined with the aid of GIS. The main conclusions are as follows: (1) Land urbanization in China shows a clear spatial difference. The greater the city scale, the faster its land urbanization. The cities with rapid land urbanization show a significant pattern of central distribution in coastal regions and a scattered distribution in the inland regions. (2) Over the last 10 years, the average quantity of land urbanization in the 656 cities was $3.82 \mathrm{~km}^{2}$, the quantity of land urbanization is differentiated by administrative grade. The average rate of land urbanization was $6.89 \%$, obviously faster than the speed of population urbanization. The rate of land urbanization reveals a pattern of differentiation between coastal and other cities. (3) In the past 10 years, the two primary land use types associated with land urbanization in China are residential and industrial, with a combined contribution rate of $52.49 \%$. The greater the scale of the city, the more significant the driving effect of industrial land. In small- and medium-scale cities of the western and central regions, the growth of residential land is the primary driver of land urbanization, while in coastal urban agglomerations and cities on important communication axes, the growth of industrial land is the main driver. (4) Overall, urban population agglomeration, industrial growth and investment are the three drivers of land urbanization in China, but cities of different scales have different drivers.
\end{abstract}

Keywords: land urbanization; urban construction land; urban area; the driving force for land urbanization; China

Received: 2014-08-18 Accepted: 2015-02-06

Foundation: National Natural Science Foundation of China, No.41401164, No.41430636; The Key Research Program of the Chinese Academy of Sciences, No.KZZD-EW-06

Author: Lin Xueqin, PhD, specialized in urban and regional development. E-mail: lin-xueqin@139.com

*Corresponding author: Wang Yang, $\mathrm{PhD}$, specialized in economic geography, and urban \& regional planning.

E-mail: wyxkwy@163.com 


\section{Introduction}

Urbanization is an important research subject in regional, urban and economic geography (Lu, 2013), with land urbanization as an important visual indicator of the process (Lv et al., 2008). In recent years in China, the process of land urbanization has been faster than population urbanization (Yao et al., 2012), and some aggressive urbanization is seen as excessively rapid and with out-of-control urban spatial expansion (Yao et al., 2011; Lu, 2007; Lu et al., 2007). During this process, land urbanization plays a more and more important supporting and restriction role (Tian, 2011). Therefore, measurement of land urbanization is as important as population urbanization. The dynamic evaluation of land urbanization focuses mainly on measuring the quantity, speed and change of land-use structures (Zhang et al., 2008; Wang and Li, 2011; Deng et al., 2009; Xu et al., 2009; Tang, 2009; Feng and Chen, 2010; Zhang et al., 2010; Dong et al., 2011). However, most of the research is based on single or limited cases, which are unfavorable for inter-city comparisons. Liu Jiyuan proposed a index to measure the land urbanization level, and an expansion index to measure the land urbanization process on the basic unit of $1 \mathrm{~km}$ raster data (Liu et al., 2012). These indices provide a good research perspective on measures of land urbanization level and process, and have important guiding and referential significance in this paper by establishing an absolute index of land urbanization.

China has a vast territory with significant regional differences (Wang et al., 2012), with corresponding differences between regions in levels of land urbanization (Chen et al., 2010; Wang, 2014), and with significant spatial agglomeration (Jiang et al., 2013). The uniform land policy nationwide can hardly conform to the varying land urbanization levels. Therefore, realizing the huge regional difference in land urbanization level in China, formulating development target and policy according to the local conditions for land urbanization are the important assurance for advancing the healthy development of new-style urbanization and the coordinated rural-urban development. Currently, the research on land urbanization in China is roughly divided into two categories, the first using a $1 \mathrm{~km} \times 1 \mathrm{~km}$ grid as the basic evaluation unit at the regional level. In this group, Liu Jiyuan et al. (2005, 2009, 2014) studied the spatial pattern and spatiotemporal evolution process of urban land (from 1990 to 2000), and urban-rural construction land (from 2000 to 2005; from 1990 to 2010), revealing a pattern and process of land urbanization in China. Tian Guangjin et al. (2003) conducted research on the spatiotemporal pattern of urban land expansion at two stages during the 1990s. The second category of research evaluates expansion of urban construction land or urban built-up areas at the city level, including urban construction land expansion of largeand medium-scale cities during the 1990s (Tan, 2004), and the expansion of urban built-up area during 1990-2010 (Wang, 2012). These construction land derived from RS interpretation inevitably include rural construction land and urban village land besides urban construction land. So these land urbanization actually is turning non-construction land into construction land. As a result, it is difficult to calculate the areas changed from rural construction land to urban construction land. In addition, turning non-construction land into rural construction land can be mistaken for 'land urbanization'. Thus, based on statistical data of land use which can distinguish urban construction land changed from rural construction land, taking urban area as the basic research unit, the study conduct an effective spatial evaluation 
of the absolute and relative speeds of land urbanization at the city level and analyzes the spatial pattern and essential feature of China's land urbanization, which are of scientific significance.

Analyzing the driving forces of land urbanization in China is the precondition and basis for comprehending its mechanism and influential factors, and is the key reference point to identify how to advance healthy land urbanization. This topic has drawn the attention of many researchers. At regional level, some of the significant themes and topics include: national macro land development strategy, fast social-economic development and rapid urbanization led to the large-scale growth of urban-rural construction land in the early 21st century (Liu et al., 2009); the growth of tertiary industry was the greatest engine for the expansion of urban construction land in China during the 1990s (Tan et al., 2004); population growth, economic development, consumption levels, technological progress, agricultural structure adjustment and intensive land use measures are the driving forces for the changes of provincial land use (Gan et al., 2004); population growth, fixed asset investment, economic growth respectively are the main driving forces for urban land expansion in different stages of development from 2003 to 2011 (Shu et al., 2013); restraint of natural conditions, adjustment of administrative divisions, economic development and population growth are the influential factors for expansion of urban land (Zhang et al., 2011); social, economic, political and spatial factors are the major drivers of spatiotemporal differences in urban land expansion in the Beijing-Tianjin-Hebei region during the 1990s (Tan et al., 2005); economic and population growth are the two major factors for the changes of urban land use in Jiangsu (Wang, 2009); and development of secondary industry is the core influence on the growth of construction land in Fujian (Wei and Chen, 2006). Research from urban case studies adds the following viewpoints for land urbanization: population growth, foreign capital investment and development of tertiary industry are the major external driving forces for the expansion of urban land in Shenzhen (Shi et al., 2000); natural environment, urban population growth, economic growth and transportation infrastructure are the main driving factors for the expansion of urban land in Changsha (Zhou and He, 2006); urban planning, market mechanisms, government development, private enterprise development, external capital investment and an excellent environment are the multi-element drivers for land expansion in Huludao (Xiu and Zhu, 2005); population growth and globalization are driving forces behind the rapid urbanization in Nanjing (Chen et al., 2014). Based on the research findings outlined above, the authors contend that analyzing major land types of urban expansion is an effective research approach to understand the driving forces underlying land urbanization in China. The factors of population, economy, industry, market, society and policy will be reflected in nine types of urban construction land. For instance, the growth of the real estate industry will be embodied in expansion of residential areas, and industrial development will affect growth of industrial land. Therefore, the proportional impact of each land-use type on urban expansion is the key point for analyzing the driving forces of land urbanization. Furthermore, since urban differentiation is significant in China, research should be carried out according to local conditions and dynamic analysis should be conducted on cities at various scales. It is of scientific significance to analyzing difference of urban scale impact on driving forces of land urbanization, and to analyzing reasons and essential characteristics.

Using urban area as the basic research unit and urban construction land area as the pri- 
mary indicator, this paper establishes the conceptual and calculation methods to determine the quantity and rate of land urbanization. The research evaluates the spatial differentiation pattern of absolute and relative urbanization processes in 658 cities in China from 2000 to 2010, analyzes the spatial distribution of cities experiencing rapid land urbanization, and explores the proportional impact and its spatial heterogeneity of major land use types in cities of different scales. The major drivers of land urbanization in China are then discussed in terms of population agglomeration, industrial growth, investment, and enhancement of urban function. Meanwhile, the research analyzes the differentiation of driving forces of land urbanization among various urban scale. It can provide a new alternative research perspective for spatial patterns, driving forces, difference characteristics of China's land urbanization.

\section{Data and methods}

\subsection{Data sources}

Urban areas of municipalities, vice-provincial cities, prefecture-level cities, and county-level cities are the designated research units, comprising a total of 656 cities. The research data mainly include urban construction land area in 2000 and 2010, subdivided into nine land-use types. The data were mainly collected from China Urban Construction Statistical Yearbook (2000, 2010). The area of urban construction land in Beijing in 2010 is sourced from China City Statistical Yearbook (2011), and the urban construction land in Shenzhen in 2000 is substituted with the urban built-up area. The data of urban drivers include urban area population, output of secondary industry, total fixed asset investment and proportion of tertiary industry in GDP in 2000 and 2010. The data of urban area population are sourced from China Urban Construction Statistical Yearbook (2000, 2010), while other data are sourced from China City Statistical Yearbook and China Regional Economic Statistical Yearbook (2001, 2011). The total number of all data items is 19,680 . The administrative divisions of a number of cities have been adjusted, and various county-level cities have incorporated into prefecture-level cities since 2000. Consequently, to ensure a consistent geographical scope of one region in different years, and to facilitate comparison of the change between the two years, administrative divisions are determined by 2010 data, and all data in 2000 are adjusted according to the administrative divisions of 2010. Finally, Taiwan Province, Hong Kong and Macao Special Administrative Regions were excluded from the study because of an absence of relevant data.

\subsection{Evaluation of land urbanization: quantity and rate of the process}

The process of land urbanization is a dynamic, involving both the scale and rate of land types transformed into urban construction land within a certain period. With reference to the “absolute urban expansion index" and "relative urban expansion index" of Liu Jiyuan (Liu et al., 2012), the area of urban land expansion represents "the quantity of land urbanization" and the speed of urban land expansion reflects the "the rate of land urbanization". The evaluation of these two metrics covers a period of one year and yields statistical calculation and tempo-spatial comparison. That is, "expansion area of urban construction land” refers to the area of land use types transformed into urban construction land within one year as an indicator of the absolute quantity of land urbanization. The "expansion speed of urban con- 
struction land" means the average annual growth rate of new urbanized areas as an indicator of the speed of land urbanization. They are respectively defined as:

$$
\begin{gathered}
L U P A=\left(U C A_{i+n}-U C A_{i}\right) / n \\
L U P R=\left[\left(U C A_{i+n} / U C A_{i}\right)^{1 / n}-1\right] \times 100 \%
\end{gathered}
$$

where LUPA is the quantity of land urbanization, $L U P R$ is the rate of land urbanization, UCA is the area of urban construction land, $i$ is the starting year, and $n$ is the number of years.

\subsection{The threshold value of land urbanization}

According to the features of population urbanization of 656 cities in China, Wang Yang et al. (2014) calculated the threshold values of the rate of land urbanization to be $3.5 \%$, $8 \%$ and $10 \%$. If the average annual growth rate of urban construction land is lower than $3.5 \%$, the city has a low rate of land urbanization, whereas the rate is $3.5 \%-8 \%$, the city has a medium rate of land urbanization, for rates of $8-10 \%$, the city has a high rate of land urbanization, and for those above $10 \%$ the city has extremely high rate of land urbanization. Similarly, the threshold values of land urbanization quantity are defined as $1 \mathrm{~km}^{2}, 5 \mathrm{~km}^{2}$ and $10 \mathrm{~km}^{2}$. In terms of these threshold values, the pace and scale of land urbanization is differentiated in this paper.

\subsection{Analysis of the hotpot area of land urbanization by using the Kernel method}

The Kernel density estimation method was used to obtain the required information from the land urbanization data. It can be applied to detect the spatial distribution of hotpot areas (Chu et al., 2012). The function can also estimate the density around sample points according to the density of points within the unit grid, and produce a smooth surface. In two-dimensional space the general form of the Kernel density function can be expressed as:

$$
\lambda(s)=\sum_{l=1}^{n} \frac{1}{\pi r^{2}} \varphi\left(d_{l s} / r\right)
$$

where $\lambda(s)$ is the Kernel density estimated location $s, r$ is the bandwidth (i.e., the search radius of the Kernel density), $n$ is the number of samples, $\varphi$ is the weight of the distance $d_{l s}$ between locations $l$ and $s$.

\subsection{Analysis of the drivers of land urbanization}

According to the preliminary analysis of influencing factors, a multivariate regression model was developed as follows:

$$
g(y)=a_{0}+a_{1} f\left(x_{1}\right)+a_{2} f\left(x_{2}\right)+\cdots+a_{n} f\left(x_{n}\right)
$$

where, $a_{0}$ is the intercept, $a_{1}, a_{2}$ and $a_{n}$ represent regression coefficients of the abovementioned influencing factors, respectively. $g(y)$ is the increased quantity of urban construction land in the study period, and $f(x)$ is the increase quantity of influential factors, which can be expressed as:

$$
\begin{gathered}
g(y)=U C A_{i+n}-U C A_{i} \\
f(x)=F_{i+n}-F_{i}
\end{gathered}
$$

where, $F_{i}$ is the absolute quantity of influential factors in $i$ year, and $n$ is the study period. 


\section{Spatial evaluation of land urbanization in China}

\subsection{City classification and spatial evaluation by scales of urban construction land}

Classification and spatial evaluation of cities by scale of urban construction land is the basis for analyzing the level of land urbanization in China. With reference to the provisions of the Urban Planning Law of the People's Republic of China on defining the urban scale, additionally, to the classification of urban built-up area by Wang Lei et al. (2012), in terms of calculating per-capita construction land as $100 \mathrm{~m}^{2}$, cities with urban construction land of less than $20 \mathrm{~km}^{2}$ are defined as small-scale cities, those with $20-50 \mathrm{~km}^{2}$ as medium-scale cities, those with 50-200 $\mathrm{km}^{2}$ as large-scale cities and those with more than $200 \mathrm{~km}^{2}$ as extraordinarily large-scale cities. Figure 1 shows that over the last decade the number of extraordinarily large-scale cities has clearly increased, from 9 in 2000 to 40 in 2010. Great changes have taken place in the spatial pattern of urban scale. The spatial distribution of cities with construction land area of $50 \mathrm{~km}^{2}$ has changed from a scattered distribution in 2000 to a coastal agglomeration distribution in 2010. In 2000, the spatial distribution of large-scale cities nationwide was relatively scattered and the distinction between coastal and inland regions not obvious. By 2010, the urban scale of the urban agglomerations of Central-South Liaoning, Beijing-Tianjin-Hebei, Shandong Peninsula, Yangtze River Delta, West-Coast-of-Straits, and Pearl River Delta, along with other eastern coastal regions had obviously increased; large-scale cities in these regions are spatially agglomerated obviously. The spatial differentiation of scales of urban construction land shows a pattern of large in coastal regions and small in inland regions. The urban scale of provincial capital cities and important prefecture-level cities in the central and western regions is also relatively large.

(a) 2000

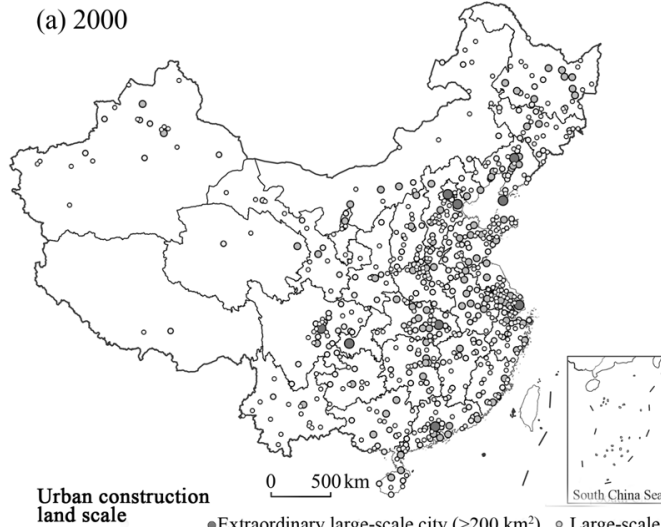

(b) 2010

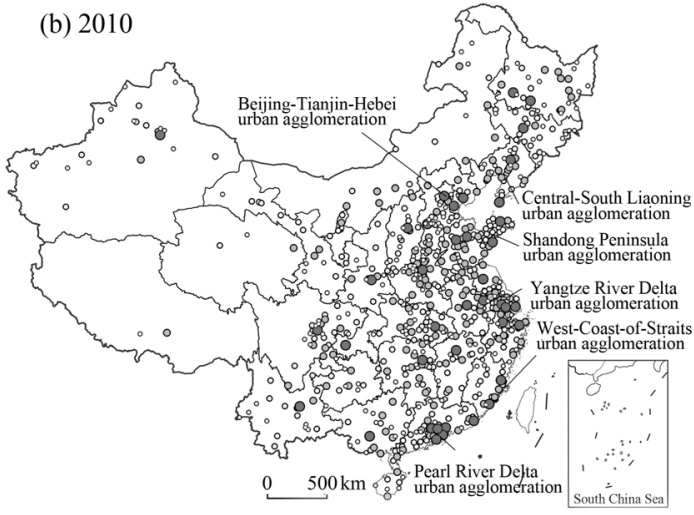

Figure 1 Spatial evaluation of urban construction land scale in China in 2000 and 2010

\subsection{Spatial differentiation of land urbanization quantity}

The average annual growth area of urban construction land in different cities in China was calculated for 2000-2010 for measuring the absolute process of land urbanization. Over the last 10 years, the average quantity of land urbanization in the 656 cities was $3.82 \mathrm{~km}^{2}$, and the larger the city the higher the quantity of land urbanization. The average quantity of land urbanization for extraordinarily large-scale cities was $26.85 \mathrm{~km}^{2}, 4.01 \mathrm{~km}^{2}$ for large-scale 
cities, $1.20 \mathrm{~km}^{2}$ for medium-scale cities and only $0.31 \mathrm{~km}^{2}$ for small-scale cities. The average annual growth of 40 extraordinarily large-scale cities was $1074.13 \mathrm{~km}^{2}$, comprising $50.57 \%$ of the total for all the cities in China. It indicates that the majority of land urbanization in China is taking place in the extraordinarily large-scale cities. Figure 2 indicates that the largest area of land urbanization is found in provincial capital cities, including municipalities and vice-provincial cities, with the average annual increase

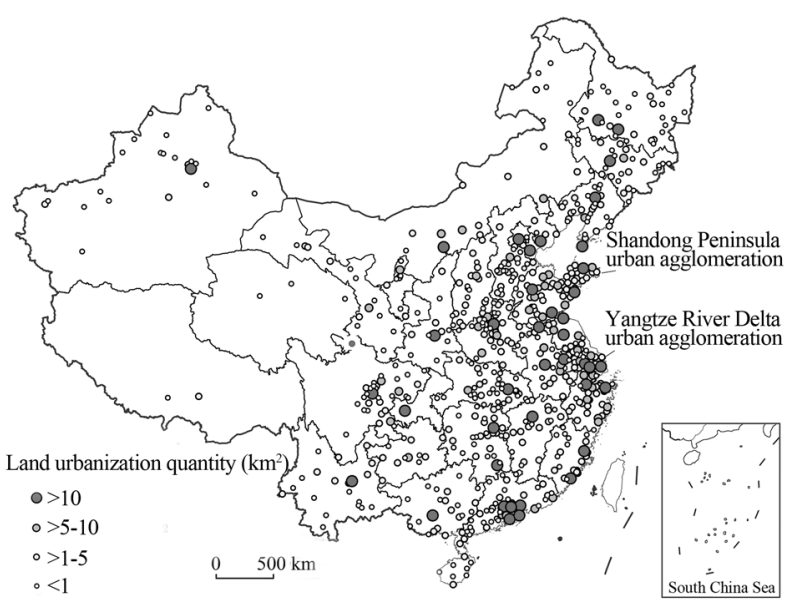

Figure 2 Spatial pattern of land urbanization quantity in Chinese 656 cities (2000-2010) generally more than $10 \mathrm{~km}^{2}$, a fact that demonstrates a clear administrative-grade differentiation. The quantity of land urbanization for some cities in the Yangtze River Delta and Shandong Peninsula urban agglomerations is also relatively large, while that for non-provincial capital cities in the central and western regions is relatively small.

The quantity of land urbanization in different administrative-grade cities is calculated and presented in Table 1. The results indicate that the higher the administrative grade the larger the quantity of land urbanization. The average annual growth in area of urban construction land is $24.05 \mathrm{~km}^{2}$ per provincial capital city, which is far higher than prefecture-level cities $\left(3.73 \mathrm{~km}^{2}\right)$ and county-level cities $\left(0.98 \mathrm{~km}^{2}\right)$. The average total construction land area of provincial capital cities has also increased rapidly from $194.75 \mathrm{~km}^{2}$ in 2000 , to $435.23 \mathrm{~km}^{2}$ in 2010 , with a growth rate of $123.49 \%$. The average construction land area of prefecture-level cities and county-level cities has grown by $92.72 \%$ and $61.41 \%$, respectively, a rate far lower than provincial capital cities.

Table 1 Land urbanization quantity for different administrative grades cities in China in 2000 and 2010

\begin{tabular}{|c|c|c|c|c|c|c|c|}
\hline \multirow{2}{*}{$\begin{array}{l}\text { Administrative } \\
\text { hierarchy }\end{array}$} & \multirow{2}{*}{$\begin{array}{l}\text { Number } \\
\text { of cities }\end{array}$} & \multicolumn{3}{|c|}{ Construction land area $\left(\mathrm{km}^{2}\right)$} & \multicolumn{2}{|c|}{$\begin{array}{l}\text { Average construc- } \\
\text { tion land area per } \\
\text { city }\left(\mathrm{km}^{2} / \text { city }\right)\end{array}$} & \multirow{2}{*}{$\begin{array}{c}\text { Average annual } \\
\text { quantity of land } \\
\text { urbanization per } \\
\text { city }\left(\mathrm{km}^{2} / \text { year·city }\right)\end{array}$} \\
\hline & & 2000 & 2010 & $\begin{array}{l}\text { Increase } \\
\text { rate (\%) }\end{array}$ & 2000 & 2010 & \\
\hline Provincial capital city & 36 & 7010.85 & 15668.31 & 123.49 & 194.75 & 435.23 & 24.05 \\
\hline Prefecture-level cities & 251 & 9982.79 & 19237.88 & 92.72 & 39.77 & 76.64 & 3.73 \\
\hline County-level cities & 369 & 5416.87 & 8743.78 & 61.41 & 14.68 & 23.70 & 0.98 \\
\hline
\end{tabular}

\subsection{Spatial differentiation of land urbanization rate}

The average annual growth rate of urban construction land in different cities in China was calculated for measuring the relative process of land urbanization. Within 10 years, the average rate of land urbanization for 656 cities in China was $6.89 \%$, obviously faster than the speed of population urbanization. The larger the scale of city, the higher the rate of its land urbanization. The average rate of land urbanization was $9.30 \%$ for extraordinarily large- 


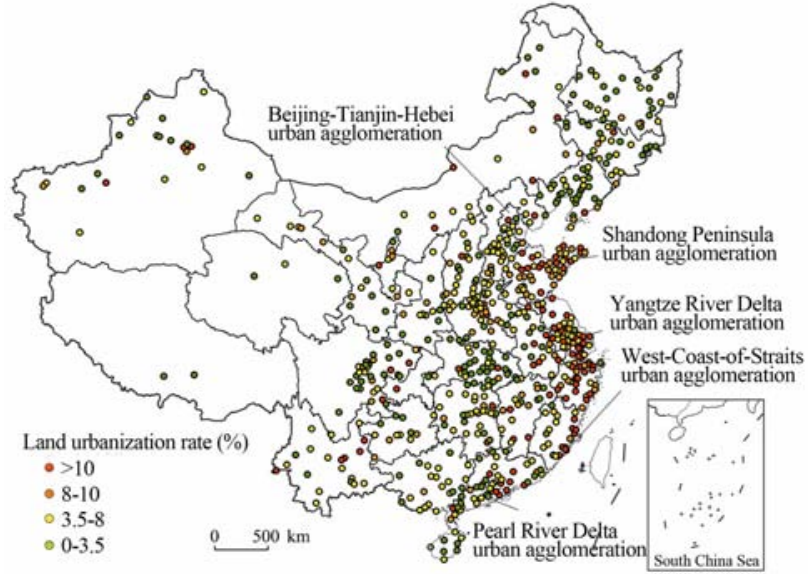

Figure 3 Spatial pattern of land urbanization rate in Chinese 656 cities (2000-2010)

sion that China's land urbanization mainly occurred in Huang-Huai-Hai Plain-Yangtze River Delta, South-East coastal areas, and Sichuan Basin. The result is consistent with our findings. For these coastal urban agglomerations, the average area of construction land per city has increased from $50.60 \mathrm{~km}^{2}$ in 2000 to $120.84 \mathrm{~km}^{2}$ in 2010 , with a rate of land urbanization as high as $9.10 \%$. The rate of land urbanization for other cities is only $5.77 \%$, thus defining a differential spatial pattern between coastal and other cities. However, the spatial difference of administrative grade is not significant. The analysis of BOX distributions of the rate of land urbanization also indicates that the distribution trend of land urbanization rate is remarkably different between coastal urban agglomerations and inland cities (Figure 4). scale cities, $6.40 \%$ for large-scale cities, 5.04\% for medium-scale cities and only $2.45 \%$ for small-scale cities. As Figure 3 illustrates, the cities with a high and extremely high rate of land urbanization are centrally distributed in the urban agglomerations of Shandong Peninsula, Yangtze River Delta, WestCoast-of-Straits, and Pearl River Delta, while the Beijing-TianjinHebei urban agglomeration shows a high rate of land urbanization. Based on $30 \mathrm{~m}$ raster data, Liu Jiyuan et al. (2014) drew a conclu-

\subsection{Spatial distribution features of cities with rapid land urbanization}

Cities with an average annual increase of urban construction land of $5 \mathrm{~km}^{2}$ are defined as cities with high quantity of land urbanization, and those with an average annual growth rate of more than $8 \%$ are defined as cities with high rate of land urbanization. With GIS technology, Kernel density function is used to explore the spatial distribution patterns of land urbanization hot areas. The search radius is set as $100,000 \mathrm{~km}^{2}$ and the Kernel density distribution chart of rapid land urbanization is thus generated (Figure 5). As Figure 5 shows, cities with a high quantity of land urbanization are centrally distributed in such provinces as Shandong, Jiangsu, Shanghai, Zhejiang and Guangdong, while other regions show a scat- 
tered distribution, and administrative-grade spatial differentiation is clear. Cities with a high rate of land urbanization present a concentrating distribution, concentrated mainly in the coastal urban agglomerations, the Central Plains urban agglomeration of inland regions, and the Hangzhou-Changsha development axis. The result is consistent with Liu Jiyuan et al. (2014) findings based on 30-m raster data. In general, the cities with rapid land urbanization show a significant pattern of central distribution in coastal regions and a scattered distribution in the inland regions.

(a) Land urbanization quantity of high-level

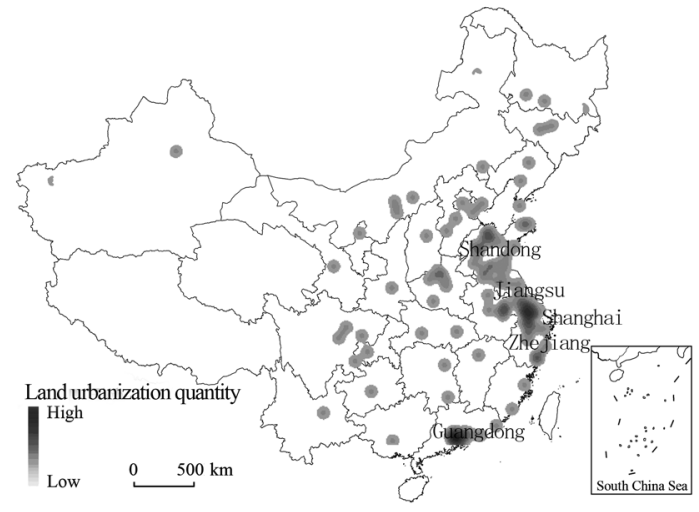

(b) Land urbanization rate of high-level

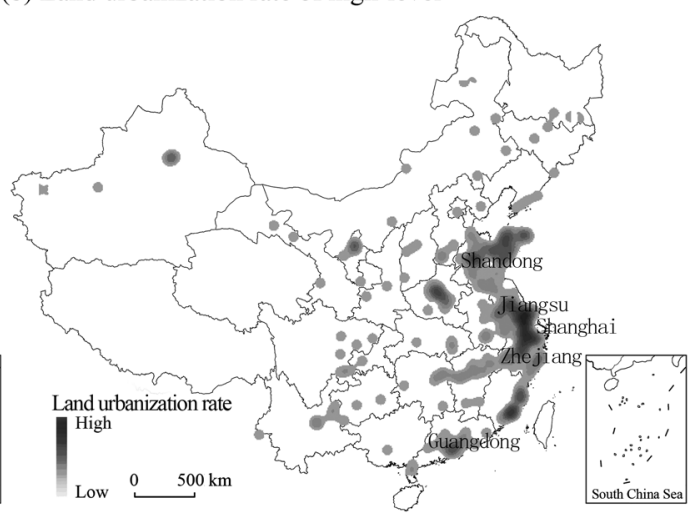

Figure 5 Kernel density distribution of cities with rapid land urbanization in China (2000-2010)

\section{Major land-use type and drivers for land urbanization}

\subsection{Land-use types for land urbanization}

Land-use type plays a leading role in land urbanization process in China and is an important precondition in analyzing land urbanization's drivers. According to the classification standard for urban construction land (GB50137-2011), there are nine land categories: residential, public facilities, industrial, warehousing, external traffic, roads and squares, green, municipal public facilities, and special purposes. Table 2 presents the proportion of different land-use types in urban construction land, and the proportion of average annual increase for various city scales.

As Table 2 shows, at a national level the proportions of residential and industrial land in urban construction land areas are the highest, at $31.20 \%$ and $21.86 \%$, respectively. The proportions of public facilities, roads and squares and green land are also relatively high, while the proportions of other land-use is relatively low. There are also differences in the land-use structure at different city scales. As the urban scale increases, the proportion of residential land gradually declines and the proportion of industrial land increases. In extraordinarily large-scale cities, the proportion of roads and squares land is relatively high, while the proportion of green land is relatively low.

Over the past decade, residential and industrial land has contributed most to the national land urbanization, with a combined contribution more than half of the total. This tends to indicate that the development of real estate and industry has driven the land urbanization process in China in the past 10 years. The contribution rate of roads and squares is $16.33 \%$, 
Table 2 Land-use structures in 2010 and land area growth structures since 2000 in various-scale cities in China

\begin{tabular}{|c|c|c|c|c|c|c|c|c|c|c|}
\hline \multirow[b]{2}{*}{ Categories } & \multirow[b]{2}{*}{ City scale } & \multicolumn{9}{|c|}{ Type of urban construction land } \\
\hline & & $\begin{array}{l}\text { Reside- } \\
\text { ntial }\end{array}$ & $\begin{array}{c}\text { Public } \\
\text { facilities }\end{array}$ & $\begin{array}{l}\text { Indus- } \\
\text { trial }\end{array}$ & $\begin{array}{l}\text { Wareho- } \\
\text { using }\end{array}$ & $\begin{array}{c}\text { Exter- } \\
\text { nal } \\
\text { traffic }\end{array}$ & $\begin{array}{c}\text { Roads } \\
\text { and } \\
\text { squares }\end{array}$ & $\begin{array}{c}\text { Municipal } \\
\text { public } \\
\text { facilities }\end{array}$ & Green & $\begin{array}{c}\text { Special } \\
\text { pur- } \\
\text { poses }\end{array}$ \\
\hline \multirow{5}{*}{$\begin{array}{l}\text { Proportion } \\
\text { of various } \\
\text { types of } \\
\text { land in } \\
\text { urban } \\
\text { construc- } \\
\text { tion land in } \\
2010 \text { (\%) }\end{array}$} & Small-scale cities & 35.31 & 12.11 & 16.95 & 3.47 & 3.83 & 11.65 & 3.53 & 11.30 & 1.85 \\
\hline & Medium-scale cities & 33.07 & 12.29 & 18.72 & 3.33 & 4.31 & 11.52 & 3.72 & 11.30 & 1.74 \\
\hline & Large-scale cities & 30.97 & 12.38 & 22.13 & 3.05 & 4.43 & 11.43 & 3.25 & 10.66 & 1.71 \\
\hline & $\begin{array}{l}\text { Extraordinarily } \\
\text { large-scale cities }\end{array}$ & 29.62 & 11.86 & 24.27 & 2.64 & 4.49 & 12.28 & 3.58 & 8.95 & 2.31 \\
\hline & Nationwide & 31.20 & 12.15 & 21.86 & 2.99 & 4.39 & 11.77 & 3.49 & 10.21 & 1.95 \\
\hline \multirow{5}{*}{$\begin{array}{l}\text { Proportion } \\
\text { of average } \\
\text { annual } \\
\text { growth } \\
\text { area 2000- } \\
\text { 2010) (\%) }\end{array}$} & Small-scale cities & 30.81 & 14.66 & 14.76 & -2.46 & -1.94 & 22.04 & 3.62 & 19.85 & -1.33 \\
\hline & Medium-scale cities & 31.46 & 12.76 & 18.20 & 0.95 & 2.10 & 15.35 & 3.55 & 15.12 & 0.51 \\
\hline & Large-scale cities & 31.41 & 14.07 & 19.67 & 1.13 & 2.40 & 15.48 & 2.97 & 12.62 & 0.26 \\
\hline & $\begin{array}{l}\text { Extraordinary } \\
\text { large-scale cities }\end{array}$ & 27.87 & 12.04 & 25.97 & 0.52 & 1.24 & 17.15 & 4.21 & 10.84 & 0.15 \\
\hline & Nationwide & 29.60 & 13.03 & 22.89 & 0.82 & 1.81 & 16.33 & 3.60 & 11.71 & 0.20 \\
\hline
\end{tabular}

Note: The above data do not include Beijing and Shanghai.

indicating land occupation of traffic construction is also relatively large. The contribution of different land-use types to land urbanization varies in different scales cities. In small-scale cities, the top three land-use types are residential, roads and squares, and green land. This indicates for small-scale cities, real estate has developed rapidly and roads and green construction are relatively fast, but industrial development is slow. Residential and industrial land is the leading types for land urbanization in large- and medium-scale cities, with the contribution of residential land far higher than that of industrial land. This reveals a strong driver in real estate development for land urbanization in these cities. However, in extraordinarily large-scale cities, the land-use types contributing most to land urbanization are residential and industrial, with their contribution rates nearly the same. This suggests that the expansion speed of residential land is lower than an expected range, while the expansion speed of industrial land is too fast, resulting in a tight supply of residential land and waste of industrial land.

From the above, it may be concluded that residential and industrial land are two major core land-use types for land urbanization in China. The contribution rates of residential and industrial land to land urbanization are calculated for different cities over the last decade (Figure 6). The threshold values of contribution rates for residential land are set from low to high at $15 \%, 25 \%, 35 \%$ and $50 \%$. Similarly the threshold values for industrial land are set at $10 \%, 15 \%, 25 \%$ and $40 \%$. As Figure 6 shows, cities with a contribution rate of residential land higher than $35 \%$ are mainly distributed in the central and western regions, and among small- and medium-scale cities. This indicates that the development of real estate is the main driver for land urbanization in these cities, and that the spatial agglomeration rule is less significant. However, the agglomeration rule for the contribution rate of industrial land is much more significant. Cities with extremely high contribution rates of industrial land are mainly concentrated in the coastal urban agglomerations, especially the Shandong Peninsula and Yangtze River Delta urban agglomerations. They are also concentrated along the important communications axes in the central and western regions, such as Harbin-Dalian, Bei- 
jing-Guangzhou, Lianyungang-Urumqi, and Shanghai-Kunming. This indicates that the land urbanization in coastal urban agglomerations and along important communications axes is mainly driven by industrial development.

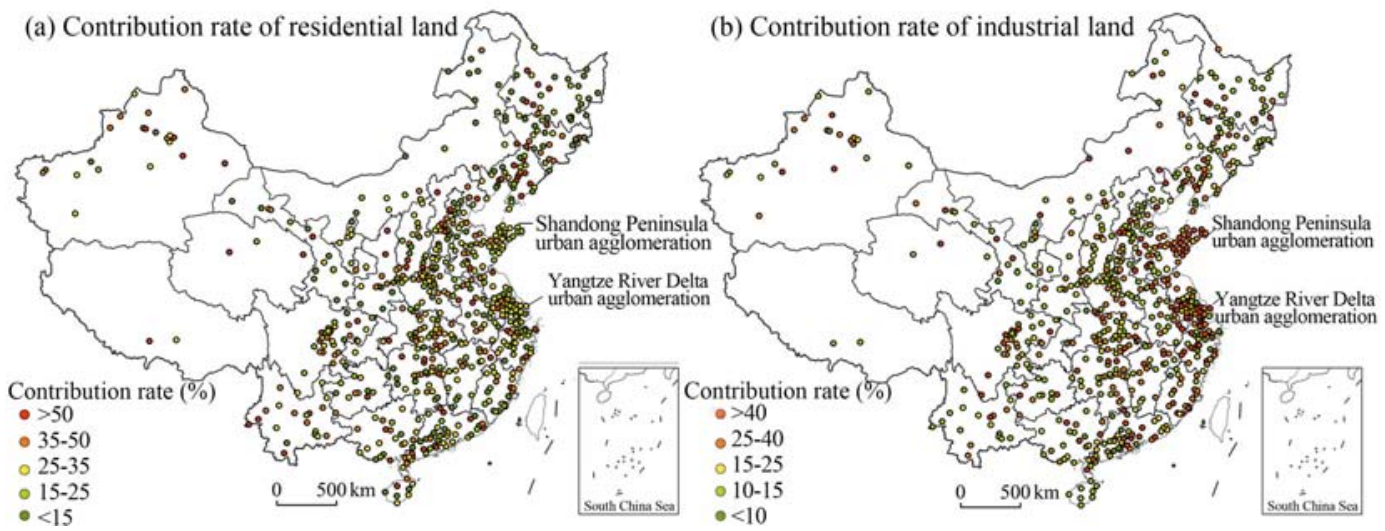

Figure 6 Contribution rate of residential and industrial land for land urbanization in China (2000-2010)

\subsection{Overall driving force for land urbanization}

Previous researches suggest that population, economy, industry, service industry, investment, and urban function etc. are the core driving forces of land urbanization in regional scale or urban scale (Tan et al., 2004; Gan et al., 2004; Tan et al., 2005; Wei and Chen, 2006; Tang, 2009; Liu et al., 2009; Zhang et al., 2011; Shu et al., 2013). According to existing research and our analysis, the drivers of land urbanization can be summarized under four main categories: urban population agglomeration, industrial growth, investment, and promotion of urban function. Population growth in cities leads to demand for housing, communication infrastructure, green space and related facilities. Cities need to constantly expand to meet the demand for living space (Zhang, 2011). Industrial growth is another important driver of land urbanization (Ding and Zhao, 2011), by creating demand for land to support production, logistical, warehousing and transport facilities. Industry also creates employment opportunities and absorbs population. Financial investment drives the demand for construction projects and enhancing urban function promotes the development of service industry and stimulates the increase of public facilities land. A regression model of land urbanization can be constructed using the growth quantity of urban construction land during 2000-2010 as the dependent variable, and the urban-area population growth, output growth of secondary industry, growth of urban fixed assets investment and proportion growth of tertiary industry output in GDP within the decade (with the constant price of 2000 as the benchmark) as the independent variables. The primary drivers of land urbanization and their differentiation among cities of varying administrative grades can then be analyzed.

According to the regression model, the goodness of fit and $d f$ value are 0.938 and 4.0 , respectively, indicating an excellent fit. The $F$ value is 1191.10 and significance level is 0.000 , indicating that the regression model is extremely significant. Table 3 shows the standard regression coefficient and its significance for four major driving factors.

As Table 3 shows, the standard regression coefficient is positive for all four factors, indicating they all have positive impact on promoting the city's land urbanization process. The significance levels of population agglomeration, industrial growth and investment are lower 
than 0.01, indicating these three factors are the primary drivers of land urbanization in China's cities. At the same time, the significance level of promoting urban function is relatively low, indicating its driving effect for land urbanization is not so significance. The regression model indicates that during 2000-2010 and with other factors unchanged, as urban population increases by 10,000, the area of urban construction land would increase by 0.704 $\mathrm{km}^{2}$, as the output of secondary industry increases by 100 million yuan (RMB), the area of urban construction land would increase by $0.033 \mathrm{~km}^{2}$, when fixed asset investment increased by 100 million yuan, the area of urban construction land would be increased by $0.045 \mathrm{~km}^{2}$.

Table 3 The parameters of regression model of land urbanization based on four major factors in China

\begin{tabular}{lccccc}
\hline \multicolumn{1}{c}{ Categories } & $\begin{array}{c}\text { Unstandardized } \\
\text { coefficients }\end{array}$ & $\begin{array}{c}\text { Standard } \\
\text { errors }\end{array}$ & $\begin{array}{c}\text { Standardized } \\
\text { coefficients }\end{array}$ & $t$ & Sig. \\
\hline Undetermined coefficient $\left(\alpha_{0}\right)$ & 1.383 & 1.275 & - & 1.085 & 0.278 \\
Population agglomeration & 0.704 & 0.021 & 0.730 & 33.657 & 0.000 \\
Industrial growth & 0.033 & 0.010 & 0.098 & 3.327 & 0.001 \\
Investment & 0.045 & 0.006 & 0.175 & 7.155 & 0.000 \\
Promotion of urban function & 13.497 & 13.125 & 0.014 & 1.028 & 0.304 \\
\hline
\end{tabular}

\subsection{Driving forces for land urbanization of different scales cities}

Cities of different scales may have different drivers of land urbanization. Regression analysis was conducted on land urbanization drivers for small-scale cities $\left(<20 \mathrm{~km}^{2}\right)$, medium-scale cities (20-50 $\left.\mathrm{km}^{2}\right)$ and large-scale cities $\left(>50 \mathrm{~km}^{2}\right)$, respectively (Table 4).

Table 4 The parameters of regression model in various-scale cities in China

\begin{tabular}{|c|c|c|c|c|c|c|c|c|c|c|c|}
\hline \multirow{2}{*}{ City scale } & \multirow{2}{*}{$\mathrm{R}$} & \multirow[t]{2}{*}{$F$} & \multirow{2}{*}{ Sig. } & \multicolumn{2}{|c|}{$\begin{array}{c}\text { Population } \\
\text { agglomeration }\end{array}$} & \multicolumn{2}{|c|}{ Industrial growth } & \multicolumn{2}{|c|}{ Investment } & \multicolumn{2}{|c|}{$\begin{array}{l}\text { Promotion of } \\
\text { urban function }\end{array}$} \\
\hline & & & & B & Sig. & B & Sig. & B & Sig. & B & Sig. \\
\hline Small-scale cities & 0.401 & 8.331 & 0.000 & $0.188^{* * *}$ & 0.000 & 0.011 & 0.353 & 0.005 & 0.613 & $5.587^{* *}$ & 0.025 \\
\hline Medium-scale cities & 0.504 & 22.587 & 0.000 & $0.357^{* * *}$ & 0.000 & 0.013 & 0.146 & $0.025^{* * *}$ & 0.009 & -2.780 & 0.590 \\
\hline Large-scale cities & 0.931 & 328.568 & 0.000 & $0.696^{* * *}$ & 0.000 & $0.033^{*}$ & 0.068 & $0.038^{* * *}$ & 0.001 & 41.415 & 0.333 \\
\hline
\end{tabular}

Note: Regression method is force regression. $*=$ significant at $10 \%$ level; $* *=$ significant at $5 \%$ level; $* * *=$ significant at $1 \%$ level.

Table 4 shows that the degree of fitness and significance of the regression model at the three city scales are relatively good, while the drivers in each case is different. Urban population agglomeration and urban function promotion are the main drivers of land urbanization in small-scale cities, population agglomeration and investment are the drivers in medium-scale cities, and in large-scale cities the drivers are population agglomeration, investment, and industrialization (the weakest of the three). In general, population agglomeration is the common driver to all three scales cities, the driver effect of investment is relatively significant in large- and medium-scale cities, urban function promotion mostly push land urbanization process in county-level cities, and industrial growth has certain driving effect for land urbanization in large-scale cities. These findings are consistent with our previous conclusions for land-use type analysis. 


\section{Conclusions}

(1) A significant spatial differentiation of land urbanization was found in China at the city level during 2000-2010. The greater the city scale, the faster its land urbanization process. Over the last 10 years, the average quantity of land urbanization in the 656 cities was 3.82 $\mathrm{km}^{2}$. The quantity of land urbanization reveals a differentiation among administrative grades. The average rate of land urbanization was $6.89 \%$, obviously faster than the speed of population urbanization. The rate of land urbanization follows a spatial pattern of differentiation between coastal and other cities, while variations of administrative grades are not significant. Cities with rapid land urbanization are centrally distributed in the coastal regions and scattered throughout the inland regions.

(2) In the last 10 years, the two primary land use types promoting land urbanization in China are residential and industrial, with a combined contribution rate of $52.49 \%$. Their expansion differs in different scales cities: in small-scale cities, the primary growth is in residential, roads and squares and green land; in large- and medium-scale cities, its growth is mainly residential land followed by industrial land; for extraordinarily large-scale cities, residential land and industrial land are both the major categories of land use growth. Generally, the larger the city scale, the higher the proportion of its industrial land. In terms of the spatial differentiation pattern, the growth of residential land is primary for land urbanization in the central and western regions, and for small- and medium-scale cities; the growth of industrial land is the core for land urbanization in coastal urban agglomerations and cities situated along important communication axes.

(3) Urban population agglomeration, industrial growth and investment are the three core drivers of land urbanization in China. Cities at different scales have varying drivers of land urbanization: in small-scale cities, population agglomeration and promotion of urban function are the major driving forces for land urbanization; in the large- and medium-scale cities, the drivers are population agglomeration and investment; and finally, the driver of industrial growth is mainly seen in large-scale cities.

\section{References}

Chen Fenggui, Zhang Hongou, Wu Qitao et al., 2010. A study on coordinate development between population urbanization and land urbanization in China. Human Geography, 25(5): 53-58. (in Chinese)

Chen Jianglong, Gao Jinlong, Xu Mengyue et al., 2014.Characteristics and mechanism of construction land expansion in Nanjing metropolitan area. Geographical Research, 33(3): 427-438. (in Chinese)

Chu Honejay, Liau Churnjung, Lin Chaohung et al., 2012. Integration of fuzzy cluster analysis and Kernel density estimation for tracking typhoon trajectories in the Taiwan region. Expert Systems with Applications, 39(10): 9451-9457.

Deng J S, Wang K, Hong Y et al., 2009. Spatiotemporal dynamics and evolution of land use change and landscape pattern in response to rapid urbanization. Landscape and Urban Planning, 92(3): 187-198.

Ding C, Zhao X, 2011. Assessment of urban spatial growth patterns in China during rapid urbanization. Chinese Economy, 44(1): 46-71.

Dong Tingxu, Qin Qiming, Wang Jianhua, 2011. Expansion mode of urban landuse in Mianyang City in the last 30 years. Geographical Research, 30(4): 667-675. (in Chinese)

Feng J, Chen Y, 2010. Spatiotemporal evolution of urban form and land-use structure in Hangzhou, China: Evidence from fractals. Environment and Planning B-Planning \& Design, 37(5): 838-856.

Gan Hong, Liu Yansui, Wang Dawei, 2004. Simulation and analysis of the human driving factors of land use type conversion. Resources Science, 26(2): 88-93. (in Chinese)

Jiang Hai, Wang Bo, Li Chengrui et al., 2013. Spatial characteristics of construction land expansion in China: A comparison analysis regarding fixed capital and non-agricultural labor. China Land Sciences, 27(5): 63-70. (in Chinese) 
Liu J Y, Kuang W H, Zhang Z X et al., 2014. Spatiotemporal characteristics, patterns, and causes of land-use changes in China since the late 1980s. Journal of Geographical Sciences, 24(2): 195-210.

Liu J Y, Zhang Q, Hu Y F, 2012. Regional differences of China's urban expansion from late 20th to early 21st century based on remote sensing information. Chinese Geographical Science, 22(1): 1-14.

Liu Jiyuan, Zhan Jinyan, Deng Xiangzheng, 2005. The spatiotemporal patterns and driving forces of urban land expansion in China during the economy reform era. Ambio, 34(6): 444-449. (in Chinese)

Liu Jiyuan, Zhang Zengxiang, Xu Xinliang et al., 2009. Spatial patterns and driving forces of land use change in China in the early 21st century. Acta Geographica Sinica, 64(12): 1411-1420. (in Chinese)

Lu Dadao, 2007. Urbanization process and spatial expansion in China. Urban Planning Forum, (4): 47-52. (in Chinese)

Lu Dadao, 2013. Contents framework of urbanization research in geography. Scientia Geographica Sinica, 33(8): 897-901. (in Chinese)

Lu Dadao, Yao Shimou, Li Guoping et al., 2007. Comprehensive analysis of the urbanization process based on China's conditions. Economic Geography, 27(6): 883-887. (in Chinese)

Lv Ping, Zhou Tao, Zhang Zhengfeng et al., 2008. Construction and application of land urbanization and corresponding measurement index system. China Land Science, 22(8): 24-28, 42. (in Chinese)

Shi Peijun, Chen Jin, Pan Yaozhong, 2000. Landuse change mechanism in Shenzhen City. Acta Geographica Sinica, 55(2): 151-160. (in Chinese)

Shu Bangrong, Zhu Jianjun, Li Yongle et al., 2013. Driving forces of urban land expansion at different stages of economic development: An empirical analysis based on provincial panel data. China Land Sciences, 27(11): 65-71. (in Chinese)

Tan M, Li X, Xie H et al., 2005. Urban land expansion and arable land loss in China: A case study of Beijing-Tianjin-Hebei region. Land Use Policy, 22(3): 187-196.

Tan Minhong, Li Xiubin, Lv Changhe, 2004. The expansion of construction land and its occupation of farmland in China's big and medium cities in 1990s. Science in China Ser. D Earth Sciences, 34(12): 1157-1165. (in Chinese)

Tang Juhua, Lv Changhe, Yang Xuchao, 2009. Analysis of driving force of urban land expansion in Suzhou-Wuxi-Changzhou region. Resources Science, 31(5): 801-806. (in Chinese)

Tian Guangjin, Liu Jiyuan, Zhuang Dafang et al., 2003. The temporal-spatial characteristics of urban land in China in 1990s by remote sensing and GIS. Quaternary Sciences, 23(4): 421-427. (in Chinese)

Tian Li, 2011.Urbanization of land in urbanization process of China: Boon or Bane? Urban Planning, (2): 11-12. (in Chinese)

Wang Lei, Li Congcong, Ying Qing et al., 2012. China's urban expansion from 1990 to 2010 with satellite romote sensing. China Science Bulletin, 57(16): 1388-1403. (in Chinese)

Wang Liping, Zhou Yingkang, Xue Junfei, 2005. Study on urban land expansion and its driving mechanism in Jiangsu province. China Land Science, 19(6): 26-29. (in Chinese)

Wang Y, Li S C, 2011. Simulating multiple class urban land-use/cover changes by RBFN-based CA model. Computers \& Geosciences, 37(2): 111-121.

Wang Yang, Fang Chuanglin, Xiu Chunliang, Liu Daqian, 2012. A new approach to measurement of regional inequality in particular directions. Chinese Geographical Science, 22(6): 705-717.

Wang Yang, Wang Shaojian, Qin Jing, 2014. Spatial evaluation and driving force of land urbanization in Chinese Cities. Geographical Research, 33(12): 2228-2238. (in Chinese)

Wei Suqiong, Chen Jianfei, 2006. A comparative research on built-land changes between Fujian and Taiwan provinces. Geographical Research, 25(1): 87-95. (in Chinese)

Xiu Chunliang, Zhu Xiangling, 2005. The spatial expansion multi-force of local central cities. Human Geography, 20(2): 9-12. (in Chinese)

Xu X G, Peng H F, Xu Q Z et al., 2009. Land changes and conflicts coordination in coastal urbanization: A case study of the Shandong Peninsula in China. Coast Manage., 37(1): 54-69.

Yao Shimou, Lu Dadao, Chen Zhenguang et al., 2012. Serious thinking about urbanization based on China's conditions. Economic Geography, 32(5): 1-6. (in Chinese)

Yao Shimou, Lu Dadao, Wang Cong et al., 2011. Urbanization in China need comprehensive science thinking: Exploration of the urbanization mode adapted to the special situation of China. Geographical Research, 30(11): 1947-1955. (in Chinese)

Zhang H Y, Uwasu M, Hara K et al., 2008. Analysis of land use changes and environmental loads during urbanization in China. Asian Archit. Build., 7(1): 109-115.

Zhang J E, Zhou Y K, Li R Q et al., 2010. Accuracy assessments and uncertainty analysis of spatially explicit modeling for land use/cover change and urbanization: A case in Beijing metropolitan area. Science China Earth Sciences, 53(2): 173-180.

Zhang Li, Lei Jun, Li Xuemei et al., 2011. The features and influence factors of urban expansion in China during 1997-2007. Progress in Geography, 30(5): 607-614. (in Chinese)

Zhou Guohua, He Yanhua, 2006. Characteristics and influencing factors of urban land expansion in Changsha. Acta Geographica Sinica, 61(11): 1171-1180. (in Chinese) 